PROCEEDINGS OF THE

AMERICAN MATHEMATICAL SOCIETY

Volume 128, Number 7, Pages 1899-1904

S 0002-9939(99)05189-8

Article electronically published on November 1, 1999

\title{
FROM HERMITE RINGS TO SYLVESTER DOMAINS
}

\author{
P. M. COHN \\ (Communicated by Ken Goodearl)
}

\begin{abstract}
The main result proved here is a new criterion for a ring to be a Sylvester domain, and so to have a universal skew field of fractions inverting all full matrices: An Hermite ring is a Sylvester domain if and only if any product of full matrices (when defined) is full. This is also shown to hold if (and only if) the set of all full matrices is lower multiplicative.

The definition of Hermite rings is weakened, but it is shown that in any case infinitely many sentences are needed.
\end{abstract}

\section{INTRODUCTION}

It is well known that the conditions for a ring to be embeddable in a skew field are quite complicated; only in the commutative case is there a simple answer: a commutative ring is embeddable in a field if and only if the ring is an integral domain. For general rings precise conditions for embeddability were only found in 1971 2, 5 and it was also shown that these conditions could not be stated as a finite set of elementary sentences [3, [5]. For special classes such as semifirs or Sylvester domains the embedding is always possible, in fact the Sylvester domains form the precise class of rings possessing a universal skew field of fractions inverting all full matrices, briefly, a fully inverting universal skew field of fractions.

Our object in this note is to prove that an Hermite ring is a Sylvester domain (and thus has a fully inverting universal skew field of fractions) if and only if the product of any two full matrices of the same order is again full. As a consequence all Hermite rings satisfying this condition have a fully inverting universal skew field of fractions. We also obtain another condition for a ring to be a Sylvester domain: the set of all full matrices must be lower multiplicative.

After a brief discussion of the different kinds of projective module types in $\$ 2$ we recall the definition of Hermite rings in $\$ 3$ and describe some of their properties, while the main theorem and its consequences occupy $\$$

I am indebted to the referee for his comments, which led to a clarification of the argument.

\section{The PRojective Module type}

In general ring theory it is often convenient to impose certain conditions on the projective modules, either to exclude pathological cases or to ensure better

Received by the editors April 17, 1998 and, in revised form, August 24, 1998.

1991 Mathematics Subject Classification. Primary 16E60; Secondary 15A30, 16D40.

(C)2000 American Mathematical Society 
behaviour. Throughout we shall assume that our rings are associative, with a unitelement which is inherited by subrings, preserved by homomorphisms and acts as identity operator on modules. Usually we also assume that our ring is non-zero, i.e. $1 \neq 0$. For any matrix $A$ we define the inner rank as follows; of the different ways of writing $A$ as a product, $A=P Q$, we choose one in which the number of rows of $Q$ is smallest. This number is called the inner rank, written $\rho(A)$. In particular, for any non-zero element $a$ the inner rank is 1 ; the inner rank of 0 is 0 , since 0 can be written as the product of two empty matrices: a $1 \times 0$ matrix times a $0 \times 1$ matrix.

For non-zero rings we have the following successively more restrictive conditions on the projective (and in particular the free) modules:

1. invariant basis number,

2. unbounded generating number,

3. weak finiteness,

4. Hermite property,

5. cancellation of projectives,

6. projective-freeness,

7. Sylvester property,

8. Semifir property.

We briefly recall the definitions (cf. [5], 0.4). 1. A ring $R$ has invariant basis number (IBN) if for all $m, n, R^{m} \cong R^{n}$ implies $m=n ; 2 . R$ has unbounded generating number (UGN) if for all $m, n$, and any $R$-module $K, R^{m} \cong R^{n} \oplus K$ implies $m \geq n ; 3$. $R$ is weakly finite if for all $n, R^{n} \cong R^{n} \oplus K$ implies $K=0 ; 4$. $R$ is Hermite if for all $m, n$, and any $R$-module $K, R^{n} \cong R^{m} \oplus K$ implies that $n \geq m$ and $K$ is free of rank $n-m ; 5 . R$ has cancellation of projectives if $R \neq 0$ and for any finitely generated projective $R$-modules $K, K^{\prime}, K \oplus R \cong K^{\prime} \oplus R \Rightarrow K \cong K^{\prime} ; 6$. $R$ is projective-free if every finitely generated projective $R$-module is free, of unique rank; 7. $R$ is a Sylvester domain if it is non-zero and for any matrix product equal to zero: $A B=0$, where $B$ has $r$ rows, we have $\rho(A)+\rho(B) \leq r ; 8$. $R$ is a semifir if every finitely generated right ideal of $R$ is free, of unique rank. More generally, $R$ is an $n$-fir if every right ideal on at most $n$ generators if free, of unique rank. It is easily verified that each of these conditions is left-right symmetric and entails the previous ones (cf. [5, 0.4, for a discussion of 1-4 and 6); moreover, all these classes are distinct (cf. 1] for 1-3). Rings beyond class 1 are usually considered part of the pathology of rings, while the rings in classes 7 and 8 are always embeddable in skew fields, in fact they have a fully inverting universal skew field of fractions. We shall be concerned here with classes 4 and 7 .

We remark that by applying Proposition 0.2.3, p. 8 of [5] to Lemma 5.7.5, p. 246 of [6] we see that coproducts of weakly finite rings over a field are again weakly finite. The same is true of IBN and UGN.

\section{HERMite RINGS}

Let $R$ be any ring; a projective $R$-module $P$ is said to be stably free if $P \oplus R^{r} \cong$ $R^{m}$, for some $r, m$. Such $P$ has an $m$-element generating set and is also called stably $m$-free. If every stably $m$-free module over $R$ is free of unique rank, for all $m \leq n, R$ is said to be $n$-Hermite. If $R$ is $n$-Hermite, for all $n$, then every stably free module is free, of unique rank, in other words, $R$ is Hermite. More precisely, if $\mathcal{H}_{n}$ denotes the class of all $n$-Hermite rings and $\mathcal{H}$ denotes the class of all Hermite 
rings, then

$$
\mathcal{H}_{1} \supset \mathcal{H}_{2} \supset \mathcal{H}_{3} \supset \cdots, \quad \bigcap \mathcal{H}_{n}=\mathcal{H}
$$

That the inclusions are strict follows from Proposition 3.2 below.

It will be helpful to have a reformulation in terms of matrices. Let $R$ be a ring and $A$ an $m \times n$ matrix over $R$; then $A$ is called completable in $R$ if either $m=n$ and $A$ is invertible or $m<n$ and for some $(n-m) \times n$ matrix $B,(A, B)^{T}$ is invertible (where, as usual, the superscript $T$ denotes the transpose) or $m>n$ and for some $m \times(m-n)$ matrix $C,(A, C)$ is invertible. Now it can be shown that $R$ is $n$-Hermite if and only if, for any $r, m \leq n$, every $r \times m$ matrix over $R$ with a right inverse satisfies $r \leq m$ and is completable (cf. [5], Theorem 0.4.1). This formulation in terms of matrices can still be weakened, as follows. A row $a=\left(a_{1}, \ldots, a_{n}\right)$ over any ring $R$ is called unimodular if there is a column $b=\left(b_{1}, \ldots, b_{n}\right)^{T}$ such that $a b=1$. The row is completable if it is so as a $1 \times n$ matrix, i.e. if it is the first row of an invertible matrix. Now we have

Proposition 3.1. A ring is $n$-Hermite if and only if every unimodular row of length at most $n$ is completable.

Proof. Clearly the condition holds for all $n$-Hermite rings. Conversely, assume that it holds and let $A, B$ be $r \times m$ and $m \times r$ matrices such that $A B=I$. Suppose first that $r \leq m \leq n$. The first row of $A$ is unimodular, hence it is completable, with inverse $P$ say, and so by replacing $A, B$ by $A P, P^{-1} B$ we reduce $A$ and $B$ to the form

$$
A=\left(\begin{array}{cc}
1 & 0 \\
a & A^{\prime}
\end{array}\right), \quad B=\left(\begin{array}{cc}
1 & 0 \\
b & B^{\prime}
\end{array}\right) .
$$

We now have $A^{\prime} B^{\prime}=I$, where $A^{\prime}$ is $(r-1) \times(m-1)$ and $B^{\prime}$ is $(m-1) \times(r-1)$, so by induction on $m$ we obtain the desired conclusion. There remains the case where $m<r$. After performing the reduction as above, we obtain the equation

$$
\left(\begin{array}{l}
A^{\prime} \\
A^{\prime \prime}
\end{array}\right)\left(B^{\prime} B^{\prime \prime}\right)=I
$$

where $A^{\prime}, B^{\prime}$ are unitriangular, i.e. they are square with ones down the main diagonal and zeros above it; hence they are invertible. Since $A^{\prime} B^{\prime \prime}=0$, it follows that $B^{\prime \prime}=0$. Likewise $A^{\prime \prime} B^{\prime}=0$ and so $A^{\prime \prime}=0$; now we have $A^{\prime \prime} B^{\prime \prime}=I$, which contradicts the fact that $A^{\prime \prime}, B^{\prime \prime}$ both vanish. Hence this case cannot occur and the proof is complete.

In order to show that the inclusions in (1) are strict, we shall for each $n$ construct an $n$-fir, hence an $n$-Hermite ring which is not $(n+1)$-Hermite. The usual argument (cf. 4], p. 344, or [5, p. 328) then shows that the class of Hermite rings, which clearly is definable by elementary sentences, cannot be defined by a finite set of sentences.

Proposition 3.2. Let $R$ be the $k$-algebra (over a commutative field $k$ ) generated by $2 n+2$ elements $a_{i}, b_{i}(i=0,1, \ldots, n)$ with defining relation $\sum a_{i} b_{i}=1$. Then $R$ is an $n$-fir, hence also $n$-Hermite, but not an $(n+1)$-Hermite ring.

Proof. Clearly $R$ is not $(n+1)$-Hermite, since $a=\left(a_{0}, a_{1}, \ldots, a_{n}\right)$ is a unimodular row that is not completable. Moreover, by regarding $R$ as a matrix reduction of the $k$-algebra generated by $a, b$ with defining relation $a b=1$ (cf. [6] 1.7) and applying 
Theorem 2.11.2, p. 148 of [5], we see that $R$ is an $n$-fir (strictly speaking, the matrix reduction produces square matrices, but the result still applies to rectangular matrices, as long as all products are defined, see the remarks after Theorem 2.11.2, p. 148f. of [5]).

\section{EMBEDDINGS IN SKEW FIELDS}

In order to study the conditions for embeddability in a skew field we shall need to refine the notion of inner rank as follows. Let $A$ be any matrix over a non-zero ring $R$ and denote by $A \oplus I_{r}$ the diagonal sum of $A$ and the $r \times r$ unit matrix. Any factorization of $A$ can also be used as a factorization of $A \oplus I_{r}$ and we thus obtain the inequality

$$
\rho\left(A \oplus I_{r}\right) \leq \rho(A)+r
$$

Hence we find that

$$
\rho(A) \geq \rho\left(A \oplus I_{1}\right)-1 \geq \rho\left(A \oplus I_{2}\right)-2 \geq \cdots .
$$

If this sequence has a limit, we denote it by $\rho^{*}(A)$ and call it the stable rank of $A$. We remark that the stable rank exists in any ring with UGN, for then the decreasing series of integers (2) is bounded below by 0 and so it has a limit. Conversely, when the stable rank exists for some matrix $A$, then the ring has UGN. For suppose that $\rho^{*}(A)=r$; then for some $n$ and all $s \geq n, \rho\left(A \oplus I_{s}\right)=r+s$. It follows that for $s \geq n$ and any $t \geq 0, \rho\left(A \oplus I_{s+t}\right)=r+s+t$, thus $r+s+t=\rho\left(A \oplus I_{s} \oplus I_{t}\right) \leq$ $\rho\left(A \oplus I_{s}\right)+\rho\left(I_{t}\right)=r+s+\rho\left(I_{t}\right)$. This shows that $\rho\left(I_{t}\right) \geq t$, which proves that $R$ has UGN.

From the definition it is clear that $\rho(A) \geq \rho^{*}(A)$; a matrix will be called full if it is square, say $n \times n$ and its inner rank is $n$. If the stable rank is $n$, the matrix is called stably full; thus every stably full matrix is full. Hermite rings have the interesting property that the converse holds; more precisely we have

Theorem 4.1. A non-zero ring $R$ is Hermite if and only if the stable rank exists and equals the inner rank.

For a proof we refer to [7] or Proposition 5.6.2, p. 262 of [5]. There it is also assumed that $R$ has UGN, but this follows from the existence of the stable rank.

We shall find that an Hermite ring is a Sylvester domain provided that it satisfies the following condition:

F. The product of full matrices of the same order is full.

In any Hermite ring satisfying condition $\mathrm{F}$ the diagonal sum of any two full matrices is necessarily full; for given full matrices $A$ and $B$, not necessarily of the same size, we can form $A \oplus I$ with the block $I$ the size of $B$ and $I \oplus B$ with the block $I$ the size of $A$; these matrices are again full, by Theorem 4.1, hence so is their product $A \oplus B$.

Theorem 4.2. Let $R$ be an Hermite ring. Then $R$ is a Sylvester domain if and only if it satisfies condition $F$ : the product of any two full matrices of the same order is full.

Proof. Let $R$ be a ring satisfying the hypotheses of Theorem 4.2 and let $A$ be an $m \times r$ and $B$ an $r \times n$ matrix such that $A B=0$. Further denote the inner ranks of $A$ and $B$ by $m^{\prime}$ and $n^{\prime}$ respectively. The earlier remark shows that the set of full matrices over $R$ is closed under diagonal sums, so we can apply Theorem 5.4.9 of 
[5] and conclude that the inner rank of any matrix over $R$ is the maximum of the orders of its full submatrices. Clearly we have $m^{\prime}, n^{\prime} \leq r$; let us keep only $m^{\prime}$ rows of $A$ including a full $m^{\prime} \times m^{\prime}$ submatrix; calling the matrix consisting of these rows $A_{1}$, we choose an $\left(r-m^{\prime}\right) \times r$ matrix $A_{2}$ such that $\left(A_{1}, A_{2}\right)^{T}$ is full. Clearly this is always possible. Similarly we can find $n^{\prime}$ columns of $B$ including a full submatrix and calling these columns $B_{1}$, we can find an $r \times\left(r-n^{\prime}\right)$ matrix $B_{2}$ such that $\left(B_{1}, B_{2}\right)$ is full. Hence their product is a full matrix with an $m^{\prime} \times n^{\prime}$ block of zeros; by Proposition 3.2.3 of [5], we have $m^{\prime}+n^{\prime} \leq r$ and it follows that $R$ is a Sylvester domain. Conversely it is clear that any Sylvester domain satisfies condition F.

As an immediate consequence of Theorem 4.2 we have

Corollary 4.3. An Hermite ring satisfying condition $F$ has a universal skew field of fractions inverting all full matrices.

A natural question at this point is to ask what happens if we merely know that the diagonal sum of full matrices is full. We shall still need to know in addition that for full matrices $A$ and $B$, the matrix

$$
\left(\begin{array}{ll}
A & 0 \\
C & B
\end{array}\right)
$$

is full, for any matrix $C$ of appropriate size. A set of matrices is said to be lower multiplicative if it contains 1 and with any matrices $A, B$ also the matrix (3), for any appropriate matrix $C$.

Suppose now that the set $\Phi$ of all full matrices is lower multiplicative. Clearly $\Phi$ is then closed under diagonal sums. Now 1 is full, as a $1 \times 1$ matrix, hence for any full matrix $A$ the diagonal sum $A \oplus I$ is again full, so all full matrices are stably full. In particular $I$ is always full, so UGN holds for $R$. Further, the product of full matrices is again full, as the following series of elementary transformations shows:

$$
\left(\begin{array}{cc}
A & 0 \\
I & B
\end{array}\right) \rightarrow\left(\begin{array}{cc}
0 & A \\
-B & I
\end{array}\right) \rightarrow\left(\begin{array}{cc}
A B & A \\
0 & I
\end{array}\right) \rightarrow\left(\begin{array}{cc}
A B & 0 \\
0 & I
\end{array}\right)
$$

To show that $R$ is an Hermite ring, we observe that since $\Phi$ is closed under products and diagonal sums, the inner rank of a matrix is the maximum of the orders of its full submatrices, by Theorem 5.4.9 of [5]. Let $A$ be a matrix of rank $r$; by elementary transformations we can ensure that the $r \times r$ submatrix in the top left-hand corner, $A_{1}$ say, is full. We have to show that the diagonal sum of $A$ with $I=I_{s}$ has rank $r+s$; this follows because we have the following series of elementary transformations leading to a matrix with a full submatrix of size $r+s$ :

$$
\left(\begin{array}{ccc}
A_{1} & A_{2} & 0 \\
A_{3} & A_{4} & 0 \\
0 & 0 & I
\end{array}\right) \rightarrow\left(\begin{array}{ccc}
A_{1} & 0 & A_{2} \\
A_{3} & 0 & A_{4} \\
0 & I & 0
\end{array}\right) \rightarrow\left(\begin{array}{ccc}
A_{1} & 0 & A_{2} \\
0 & I & 0 \\
A_{3} & 0 & A_{4}
\end{array}\right) .
$$

This shows that the stable rank of $A$ equals its rank, hence $R$ is an Hermite ring, by Theorem 4.1 We can now invoke Theorem 4.2 to conclude that $R$ is a Sylvester domain and so obtain a result which improves Proposition 7.5.7 (i) of [5] (which assumed also that $R_{\Phi} \neq 0$ ):

Theorem 4.4. A ring is a Sylvester domain if and only if the set of all full matrices is lower multiplicative. Hence such a ring has a fully inverting universal skew field of fractions. 


\section{REFERENCES}

1. P. M. Cohn, Some remarks on the invariant basis property, Topology 5 (1966), 215-228. MR 33:5676

2. P. M. Cohn, Un critère d'immersibilité d'un anneau dans un corps gauche, Comptes Rendus Acad. Sci. (Paris) Sér. A 272 (1971), 1442-1444.

3. P. M. Cohn, The class of rings embeddable in skew fields, Bull. London Math. Soc., 6 (1974), 147-148. MR 51:3216

4. P. M. Cohn, Universal Algebra (revised and enlarged edition), D. Reidel, Dordrecht-Boston, 1981.

5. P. M. Cohn, Free Rings and their Relations, 2nd edition, LMS Monographs No. 19, Academic Press, London and Orlando, 1985. MR 87e:16006

6. P. M. Cohn, Skew Fields, Theory of General Division Rings, Encyclopedia of Mathematics and its Applications, Vol. 57, Cambridge University Press, 1995. MR 97d:12003

7. P. M. Cohn and A. H. Schofield, On the law of nullity, Math. Proc. Camb. Phil. Soc. 91 (1982), 357-374. MR 83h:16004

University College London, Gower Street, London WC1E 6BT, United Kingdom

E-mail address: pmc@math.ucl.ac.uk 